ORIGINAL ARTICLE

\title{
Quality control parameters on a large dataset of regionally dissected human control brains for whole genome expression studies
}

\author{
Daniah Trabzuni,* Mina Ryten, ${ }^{*}$ Robert Walker, $\uparrow$ Colin Smith, $\dagger$ \\ Sabaena Imran,* Adaikalavan Ramasamy, $\ddagger$ Michael E. Weale $\ddagger$ and \\ John Hardy* \\ *Reta Lilla Weston Laboratories and Departments of Molecular Neuroscience, UCL Institute of \\ Neurology, London, UK \\ $\dagger$ MRC Sudden Death Brain Bank Project, Department of Neuropathology, University of \\ Edinburgh,Edinburgh, , UK \\ ${ }^{\ddagger}$ Department of Medical \& Molecular Genetics, King’s College London, Guy’s Hospital, London, UK
}

\section{Abstract}

We are building an open-access database of regional human brain expression designed to allow the genome-wide assessment of genetic variability on expression. Array and RNA sequencing technologies make assessment of genomewide expression possible. Human brain tissue is a challenging source for this work because it can only be obtained several and variable hours post-mortem and after varying agonal states. These variables alter RNA integrity in a complex manner. In this report, we assess the effect of post-mortem delay, agonal state and age on gene expression, and the utility of $\mathrm{pH}$ and RNA integrity number as predictors of gene expression as measured on 1266 Affymetrix Exon Arrays. We assessed the accuracy of the array data using QuantiGene, as an independent non-PCR-based method. These quality control parameters will allow database users to assess data accuracy. We report that within the parameters of this study post-mortem delay, agonal state and age have little impact on array quality, array data are robust to variable RNA integrity, and brain $\mathrm{pH}$ has only a small effect on array performance. QuantiGene gave very similar expression profiles as array data. This study is the first step in our initiative to make human, regional brain expression freely available.

Keywords: brain $\mathrm{pH}$, microarray validation, post-mortem human brain, QTL, RIN, RNA.

J. Neurochem. (2011) 119, 275-282.
Microarray analysis and RNA sequencing of the transciptome in post-mortem human brain tissue is a vital tool in investigating the complex genetic mechanisms involved in neurodegenerative and psychiatric disorders (Gilbert et al. 1981; Glanzer et al. 2004; Myers et al. 2007). However, there are many variables which influence the RNA integrity in post-mortem human brain tissues which need to be accounted for such data to be highly reliable (SajdelSulkowska et al. 1988; Burke et al. 1991; Glasel 1995; Imbeaud et al. 2005; Schroeder et al. 2006; Birdsill et al. 2010; Durrenberger et al. 2010).

It is important to have a reliable and stable method to assess the quality of RNA samples generated from precious heterogeneous tissues, especially from small anatomical regions, such as the substantia nigra and hypothalamus. The most widespread measure for estimating the integrity of
RNA samples at present is the RNA Integrity Number (RIN) as calculated by the Agilent 2100 Bioanalyzer for electrophoresis (Agilent Technologies UK Ltd, Edinburgh, UK).

Received May 24, 2011; revised manuscript received July 17, 2011; accepted August 12, 2011.

Address correspondence and reprint requests to John Hardy, Reta Lilla Weston Laboratories and Departments of Molecular Neuroscience, UCL Institute of Neurology, Queen Square, London WC1N 3BG, UK. E-mail: j.hardy@ion.ucl.ac.uk

Abbreviations used: CRBL, cerebellum; eQTL, expression QTL; LRRK2, leucine-rich repeat kinase 2; MAPT, microtubule-associated protein tau; MRC, Medical Research Council; OCTX, occipital cortex; PMI, post-mortem interval; PUTM, putamen; QG, QuantiGene; RIN, RNA Integrity Number; SCN8A, sodium channel, voltage-gated, type VIII, alpha subunit; sQTL, splicing QTL; SHRI, Sun Health Research Institute; WHMT, white matter. 
The RIN ranges from undetectable to ten, with undetectable being completely degraded and 10 being the most intact RNA. The calculation of RIN value is largely based on ribosomal RNA separation although this measure has been shown to be inconsistent (Imbeaud et al. 2005; Schroeder et al. 2006; Sherwood et al. 2011).

We are building a publicly accessible database of regional human brain expression, the UK Human Brain Expression Consortium, to allow the assessment of the genetic variability in gene expression (expression quantitative trait loci, eQTLs) and splicing (splicing quantitative trait loci, sQTL) as well as detailed genome-wide expression analysis (Hardy et al. 2009). To that end, we are collecting a large series of control human brain tissues (originating from $\sim 130$ individuals) in which we are dissecting 13 different CNS areas: prefrontal cortex Brodmann areas 9 and 46, parietal cortex Brodmann areas 3,1, and 2, occipital cortex (OCTX) Brodmann areas 17, temporal cortex Brodmann areas 21,41 and 42, central white matter (WHMT) below Brodmann areas 39 and 40, hippocampus, thalamus, hypothalamus, putamen (PUTM), cerebellum (CRBL), substania nigra, medulla and spinal cord. From each individual brain, we isolated DNA for whole genome genotyping analysis and from each region we isolated RNA for whole transcriptome exon array analysis. This resulted in a total of 1266 RNA samples analysed on Affymetrix Exon arrays and represents by far the largest single CNS expression dataset at present. For this quality control study, we focused on analysing the factors that affected the reliability of the RNA samples.

In this study, we assess: (i) the effects of brain bank, age, gender, cause of death, region, post-mortem delay and brain pH on RIN-based RNA quality, and, (ii) the effects of RNA quality on the performance quality of the array experiment, which was measured by a reliable and widely used parameter, present call $(\% \mathrm{P}) . \% \mathrm{P}$ is the percentage of probe sets with signal detection above background noise. We examine the effects of RNA quality on the cDNA preparation and cRNA production as part of the quality control of the array experiment, and finally we confirm the reproducibility of array data using QuantiGene (QG), a novel, PCR-independent platform (Canales et al. 2006; Arikawa et al. 2008; Hall et al. 2011).

\section{Materials and methods}

Human post-mortem brain tissue collection and dissection Brain tissues originating from 101 control Caucasian individuals were collected by the Medical Research Council (MRC) Sudden Death Brain and Tissue Bank (Edinburgh, UK; Millar et al. 2007). The bodies were stored refrigerated and were brought up to the PM suite just prior to the start of the autopsy. Each postmortem brain dissection was carried out in the same way. The whole brain was removed within $15 \mathrm{~min}$ of the body as fresh tissue. The brain was immediately cut into coronal slices and the various anatomical regions of interest were immediately sampled. Furthermore, the samples once removed from the coronal slices were placed in sealed containers which in turn were placed on cool blocks (chilled to -20C) and stored within an insulated box. The samples were dissected into various size pieces approximately 250-500 mg and were placed in tubes which were immediately snap frozen in liquid nitrogen. The post-mortem interval (PMI) was calculated from the time of death to the time of removal of the brain from the skull. The total number of samples processed from this source was 1842 as some CNS regions were not available. In all cases, control status was confirmed by histology performed on sections prepared from paraffin embedded brain blocks and the diagnosis was determined by a consultant neuropathologist. Detailed phenotypic information is described in Table 1.

An additional 36 brains originating from neuropathologically confirmed control Caucasian individuals were collected by the Sun Health Research Institute (SHRI) an affiliate of Sun Health Corporation, USA (Beach et al. 2008). In this case, whole brains were removed as fresh tissue at autopsy and brain coronal slices were frozen. Anatomical regions of interest were sampled from brain coronal slices on dry ice. The time interval from the removal of the brain at the mortuary to the completion of the dissection and placement of samples within the storage freezer ranged from 2.5 to $4 \mathrm{~h}$. The parietal cortex, hypothalamus and spinal cord regions were not available for these samples. The total number of samples processed was 476 as again, some regions were not available from some brains. Detailed phenotypic information is described in Table 1.

All samples from both sites had fully informed consent for retrieval and were authorised for ethically approved scientific investigation (Research Ethics Committee number 10/H0716/ $3)$.

Table 1 Demographics of the samples studied. Values (range, mean) for variables in the cohort from both MRC-UK and SHRI-USA data set separately and joined. Of note, however, because of the different practices of the two tissue resources, there is no overlap in the PMIs between the MRC-UK (long PMI) and SHRI-USA (short PMI)

\begin{tabular}{|c|c|c|c|c|c|c|c|c|c|c|c|}
\hline \multirow[b]{2}{*}{ Brain bank } & \multirow[b]{2}{*}{ Individuals } & \multicolumn{2}{|l|}{ Sex } & \multicolumn{2}{|c|}{ Age/year } & \multicolumn{2}{|l|}{ Brain $\mathrm{pH}$} & \multicolumn{2}{|l|}{ PMI (h) } & \multicolumn{2}{|l|}{ RIN no. } \\
\hline & & Male & Female & Range & Mean & Range & Mean & Range & Mean & Range & Mean \\
\hline MRC-UK & 101 & 78 & 23 & $16-83$ & 50.4 & $5.42-6.31$ & 6.3 & $28-114$ & 52.2 & $1-8.5$ & 4 \\
\hline SHRI-USA & 36 & 24 & 12 & 53-102 & 80 & $\mathrm{NA}$ & NA & $1-5.5$ & 2.6 & $1-8$ & 3.6 \\
\hline UK + USA & 137 & 102 & 35 & $16-102$ & 59 & $5.42-6.31$ & 6.3 & $1-114$ & 43.7 & $1-8.5$ & 3.85 \\
\hline
\end{tabular}


Post-mortem determination of brain $\mathrm{pH}$

Brain $\mathrm{pH}$ from the MRC-UK samples was recorded from multiple regions using a Hanna HI8424 hand-held $\mathrm{pH}$ meter with a glass bodied electrode (Fisher Scientific, Loughborough, UK). Consistent with previous studies, the $\mathrm{pH}$ did not vary between different brain regions (Stan et al. 2006; Monoranu et al. 2009). We therefore used a single $\mathrm{pH}$ value that measured from the lateral ventricle. The electrode was inserted into the lateral ventricle after the brain was coronally cut behind the mamillary bodies. The $\mathrm{pH}$ is influenced by that of the tissue comprising the wall of the lateral ventricle and to a lesser extent by the $\mathrm{pH}$ of the remaining CSF fluid within the lateral ventricle. The $\mathrm{pH}$ was not measured in the SHRI-USA samples.

\section{RNA extraction}

Total RNA was isolated from human post-mortem brain tissues based on the single-step method of RNA isolation (Chomczynski and Sacchi 1987) using the miRNeasy 96 kit (Qiagen, Crawley, UK). Brain tissues (50-100 mg) were collected and weighed in RNase-free 96-well plates. A minimum of one extraction was performed from each tissue sample. All steps were performed on dry ice prior to the addition of the QIAzol ${ }^{\circledast}$ Reagent. All samples were homogenised in $4^{\circ} \mathrm{C}$ using the TissueLyser II (Retsch, Castleford, UK) for $4-5 \mathrm{~min}$ at $30 \mathrm{~Hz}$ in $800 \mu \mathrm{L}$ of QIAzol with the addition of two 3-mm stainless steel beads. In this step, the solution was homogenised until no large pieces remained.

The homogenised tissue samples were incubated at room temperature $\left(15-25^{\circ} \mathrm{C}\right)$ for $5 \mathrm{~min}$. After incubation, $160 \mu \mathrm{L}$ of chloroform $\left(\mathrm{CHCl}_{3}\right)$ (Sigma-Aldrich, Gillingham, UK) was added, the plates were mixed vigorously using the TissueLyser II for $30 \mathrm{~s}$ at $30 \mathrm{~Hz}$. Plates were incubated at room temperature $\left(15-25^{\circ} \mathrm{C}\right)$ for $7-$ $10 \mathrm{~min}$. The plates were centrifuged at $6000 \mathrm{~g}$ for $45 \mathrm{~min}$ at $4^{\circ} \mathrm{C}$.

The aqueous phase (upper layer, with approximately $60 \%$ of the total volume after the QIAzol was added) was transferred to fresh RNase-free 96 -well plates. RNA was precipitated by adding $800 \mu \mathrm{L}$ of $100 \%$ ethanol to the aqueous phase (1.5 volume of the aqueous phase), followed by mixing the samples. The samples were then transferred into the RNeasy 96-well plates to allow the RNA in the solution to bind to the membrane by centrifugation.

The plates were centrifuged at $5600 \mathrm{~g}$ for $2 \mathrm{~min}$ at $21^{\circ} \mathrm{C}$, and the supernatant was discarded. Samples were washed to remove salt traces and impurities by adding $600 \mu \mathrm{L}$ of washing buffer and centrifuging plates for 2 min. Washes were performed 3-4 times. Finally, total RNA was eluted in $65 \mu \mathrm{L}$ of pre-heated RNase-free water $\left(50^{\circ} \mathrm{C}\right)$.

The concentration and purity of each RNA sample was assessed using the NanoDrop ND-1000 Spectrophotometer V3.3.0. The concentration of each sample was calculated, together with the ratio of absorbance at $260 \mathrm{~nm} / 280 \mathrm{~nm}$ and $260 \mathrm{~nm} / 230 \mathrm{~nm}$.

After purification all RNA samples were applied to a RNA 6000 Nano-LabChip and analysed using the Agilent 2100 Bioanalyzer (Agilent Technologies UK Ltd) to obtain the RIN values. RINs and total RNA electropherograms were calculated by the 2100 Expert Software (Agilent Technologies UK Ltd). Further assessment of the RIN was performed by checking each electropherogram visually.

Expression profiling using Affymetrix GeneChip Human Exon 1.0 ST Arrays

Expression profiling on the Affymetrix GeneChip ${ }^{\circledR}$ Human Exon 1.0 ST Arrays (Affymetrix, High Wycombe, UK) was performed at
AROS Applied Biotechnology AS company laboratories (http:// www.arosab.com/).

Total RNA (200 ng) was used as starting material for the cDNA preparation. First and second strand cDNA synthesis, the in vitro transcription reaction to generate cRNA and the second round of cDNA synthesis was performed using the Ambion ${ }^{\circledR}$ WT Expression Kit according to the manufacturer's instructions. Biotin labelling was performed using the Terminal Labeling Kit (Affymetrix) according to the manufacturer's instructions. Following the in vitro transcription reaction, the unincorporated nucleotides were removed using RNeasy columns (Qiagen).

Prior to hybridisation, the fragmented cDNA was heated to $95^{\circ} \mathrm{C}$ for $5 \mathrm{~min}$ and subsequently to $45^{\circ} \mathrm{C}$ for $5 \mathrm{~min}$ before loading onto the Affymetrix Human Exon 1.0 ST array cartridge. The array cartridge was then incubated for $16 \mathrm{~h}$ at $45^{\circ} \mathrm{C}$ at constant rotation (60 rpm). The washing and staining procedure was performed in the Affymetrix Fluidics Station 450. The array was exposed to 10 washes in $6 \times$ SSPE-T at $250^{\circ} \mathrm{C}$ followed by four washes in $0.5 \times$ SSPE-T at $50^{\circ} \mathrm{C}$. The biotinylated cRNA was stained with a streptavidin-phycoerythrin conjugate, final concentration $2 \mathrm{mg} / \mathrm{mL}$ (Affymetrix) in $6 \times$ saline sodium phosphate EDTA buffer with $0.01 \%$ Tween-20 (SSPE-T) for $30 \mathrm{~min}$ at $25^{\circ} \mathrm{C}$ followed by 10 washes in $6 \times \mathrm{SSPE}-\mathrm{T}$ at $25^{\circ} \mathrm{C}$. This was followed by an antibody amplification step using normal goat $\mathrm{IgG}$ as blocking reagent, final concentration $0.1 \mathrm{mg} / \mathrm{mL}$ (Affymetrix) and biotinylated anti-streptavidin antibody (goat), final concentration $3 \mathrm{mg} / \mathrm{mL}$ (Affymetrix). This was followed by a staining step with a streptavidinphycoerythrin conjugate, final concentration $2 \mathrm{mg} / \mathrm{mL}$ (Affymetrix, UK) in $6 \times \mathrm{SSPE}-\mathrm{T}$ for $30 \mathrm{~min}$ at $25^{\circ} \mathrm{C}$ and 10 washes in $6 \times \mathrm{SSPE}-\mathrm{T}$ at $25^{\circ} \mathrm{C}$. The arrays were scanned at $560 \mathrm{~nm}$ using a confocal laserscanning microscope (GeneChip ${ }^{\circledR}$ Scanner 3000 7G).

\section{Array quality control}

The Expression Console ${ }^{\mathrm{TM}}$ (EC) software version 1.1 (Affymetrix) was used to evaluate the performance quality of the arrays including the labelling, hybridisation, scanning and background signals by Probe Set summarisation and CHP file generation using Robust Multichip Analysis. The quality assessment was performed by generating different parameters for all the probesets analysed by EC; $\% \mathrm{P}$ is the main parameter that is used for the array quality in this study.

In addition, cDNA and cRNA Agilent 2100 Bioanalyzer profiles were generated for samples with wide range of RIN numbers (RINs from 2 to7) to assess the cDNA preparation and cRNA production nucleotide lengths from RNA samples with different levels of degradation.

\section{Exon array data analysis}

All arrays were pre-processed using Robust Multichip Analysis quantile normalisation with GC background correction in Partek's Genomics Suite v6.6 (Partek Inc., St. Louis, USA) (Lockstone 2011). After re-mapping the Affymetrix probe sets onto human Ref Seq build 19 (GRCh37) as documented in the most recent Netaffx annotation file (HuEx-1_0-st-v2 Probeset Annotations, Release 31), we restricted analysis to 308,717 probe sets that had a gene annotation and contained at least three probes with unique hybridisation. The gene-level expression was calculated for 27000 genes by the median of probe sets corresponding to each gene. 
Array validation using direct RNA quantification with branched DNA, QuantiGene ${ }^{\circledR} 2.0$ Assay

CRBL, OCTX, PUTM and WHMT samples from 12 individuals were analysed using the QG platform for validation of exon array results. We focused on three target genes for validation, leucine-rich repeat kinase 2 (LRRK2), sodium channel, voltage-gated, type VIII, alpha subunit (SCN8A), and microtubule-associated protein tau (MAPT). We selected ribosomal protein, large, $\mathrm{P} 0$ and ubiquitin $\mathrm{C}$ as housekeeping genes to normalise the target genes as they showed relatively low variability in expression levels (i.e. low coefficient of variation) in all brain regions in our dataset. The approach to the selection of reference genes is explained in previous studies (de Jonge et al. 2007; Coulson et al. 2008).In addition, a recent study confirms the efficiency of using this approach in selecting housekeeping genes to normalise in different tissues (Chervoneva et al. 2010). A summary of the QG probes used for analysis of all five genes is provided in Table 2.

QuantiGene 2.0 Reagent System was used and the protocol in the QuantiGene 2.0 Reagent System User Manual was followed with the exception of the substrate step. Lumigen ${ }^{\circledR}$ Lumi-Phos ${ }^{\circledR}$ Plus and $10 \%$ lithium lauryl sulfate was used instead of Lumigen ${ }^{\circledR}$ APS-5 substrate. A Biotek ELx 405 select plate washer was used for all of the wash steps in the assay. The QG 2.0 plates were then read on a Molecular Devices LMax luminometer with the plate incubator set to $45^{\circ} \mathrm{C}$ to maintain the temperature of the Lumigen ${ }^{\circledR}$ Lumi-Phos ${ }^{\circledR}$ Plus substrate. In total, 13 QG 2.0 plates were run to cover all target genes and the house keeping genes. Each house keeping gene ribosomal protein, large, P0 and ubiquitin $\mathrm{C}$ was loaded in duplicates at $12.5 \mathrm{ng} / \mathrm{well}$. In addition, target genes (LRRK2, SCN8A and MAPT) were loaded in duplicates at $75 \mathrm{ng} / \mathrm{well}$.

\section{Statistical analysis}

Linear mixed regression analyses were performed to investigate the relationship between age, gender, cause of death, region, PMI, $\mathrm{pH}$, RIN and present call (\%P). Statistical analyses were conducted using Partek ${ }^{\circledR}$ Genomics Suite ${ }^{\mathrm{TM}}$ version 6.6 and PASW statistic version 18 software. We assessed explanatory power in a forwards stepwise manner, by examining the increase in variation explained when a new covariate or set of covariates were added to the existing model, together with a $p$-value for that increase.

\section{Results}

This study involved the analysis of brain tissue originating from 137 individuals. Since we were not able to obtain all 13

Table 2 QuantiGene probes used to perform array validation

\begin{tabular}{lll}
\hline Gene & Catalogue no. & Designed to hybridise to \\
\hline LRRK2 & 83322 SA-26988 & Human LRRK2 \\
SCN8A & 83324 SA-17320 & $\begin{array}{l}\text { Human SCN8A transcript } \\
\text { variants 1 and 2 }\end{array}$ \\
MAPT & 81849 SA-15486 & Human MAPT, all six variants \\
UBC & 80041 SA-10061 & Human UBC \\
RPLP0 & 81152 SA-11148 & Human RPLP0 transcript \\
& & variants 1 and 2 \\
\hline
\end{tabular}

brain regions of interest from each individual, a total of 1302 tissue blocks were available. In the majority of cases (870 of 1302 tissue blocks) multiple RNA extractions were performed, resulting in 2318 RNA samples in total. Each of these RNA samples was assessed for RNA integrity as measured by RIN. A single RNA extraction from each tissue sample was selected on the basis of Agilent 2100 Bioanalyzer profile for downstream analysis using the Affymetrix Exon arrays (Fig. 1).

The effect of age, gender, cause of death, region, PMI and brain $\mathrm{pH}$ on RIN-based RNA quality

We assessed the dependence of RIN values on the other covariates available to us. Our focus was on the explanatory power of our available covariates on the RIN for each RNA extraction (2318 RNA samples). In this study, we assessed 2318 RNA samples. The calculated RIN ranged from 1 to 8.5 with a mean of 3.85 (Table 1). Forty-three per cent of our samples had RIN values of $<3$. We found that $33 \%$ of the variation in RIN was explained by differences among tissue blocks (adjusted $R^{2}$ measure), which set an upper limit for the explanatory power of our covariates, which all act at a between-tissue-sample level. Sixteen per cent of the variation in RIN was explained by individual-level differences.

Outside of $\mathrm{pH}$ and PMI, which were analysed separately, the most important covariates were brain region (explained $9.2 \%$ of the variation in RIN number, $p=1.7 \times 10^{-42}$ ), age (explained an additional $1.1 \%, p=4.1 \times 10^{-05}$ ) and cause of death (explained an additional 1.9\%, $p=0.022$ ). Brain bank and gender together explained an additional $0.4 \%$ of the variation.

$\mathrm{pH}$ and PMI were investigated separately, because $\mathrm{pH}$ was not measured in the SHRI-USA dataset and because the range of PMIs from the two brain banks did not overlap.

$\mathrm{pH}$ explained $2.1 \%$ of the variation in the RIN number from the MRC-UK brain bank $\left(p=1.0 \times 10^{-4}\right)$ (Fig. 2a).We note that six individuals had very low $\mathrm{pH}<5.90$ and when excluded, the correlation was no longer significant (Fig. 2b).

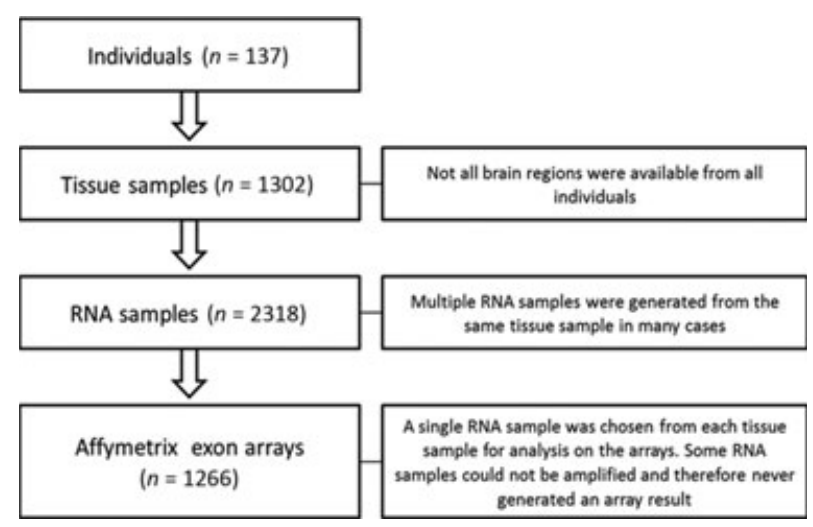

Fig. 1 Description of the data used in the analysis. 

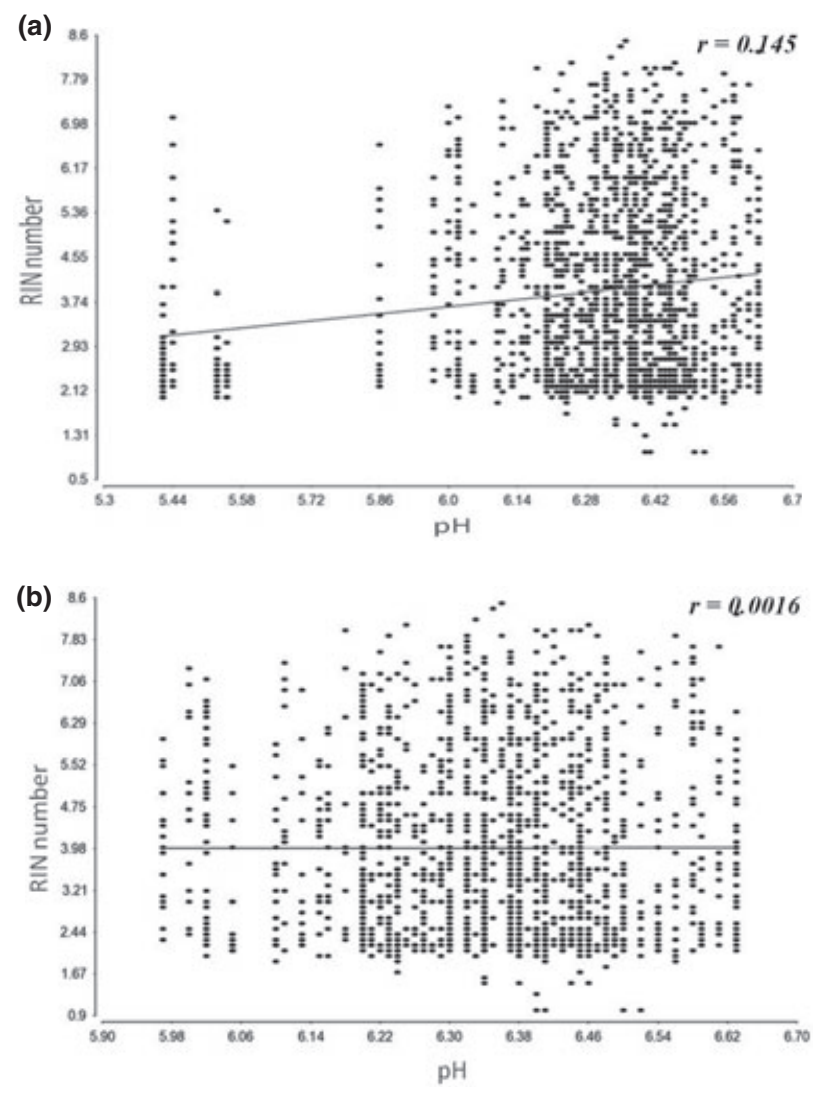

Fig. 2 (a) Scatter plot for total RNA samples with linear regression line of $\mathrm{RIN}$ numbers for $\mathrm{pH}$. Plot shows the effect of $\mathrm{pH}$ on $\mathrm{RIN}$ number for RNA samples isolated from 13 region of control brain tissue. Test $p$-values is test $p$-value $=1.0 \times 10^{-4}, r$-value $=0.145$. Including the low $\mathrm{pH}$ values of $<5.9$. (b) Scatter plot for total RNA samples with linear regression line of RIN numbers for $\mathrm{pH}$. Plot shows the effect of $\mathrm{pH}$ on RIN number for RNA samples isolated from 13 region of control brain tissue. No significant correlation was obtained with $r$-value $=0.0016$ and $p$-value $=0.82$. Samples with low $\mathrm{pH}$ value of $<5.9$ were excluded.

The effect of PMI on RIN differed between the MRC-UK and SHRI-USA datasets. There was no significant correlation between PMI and RIN in the MRC-UK dataset, which had PMIs ranging from 28 to $114 \mathrm{~h}$. In contrast, PMI explained $8.4 \%$ of the variation in RIN in the SHRI-USA dataset ( $p=0.0053$ ), which had PMIs ranging from 1 to $5.5 \mathrm{~h}$. Longer PMI was associated with a higher RIN in this data set, a counterintuitive result which might represent confounding with some unmeasured variables in the study.

The effects of age, gender, cause of death, region, PMI, brain $\mathrm{pH}$ and RIN-based RNA quality on array performance (\%P, cDNA and cRNA profile)

We assessed the dependence of RNA array performance quality on RIN-based RNA quality and the other covariates available to us. A systematic quality control check of the arrays was performed using Expression Console ${ }^{\mathrm{TM}}$ software. This software produces a number of array quality measures. The most reliable and widely used parameter is the present call (\%P) (Tomita et al. 2004; Weis et al. 2007). The percent present call is the percentage of probe sets with signal detection above background probe level $p$-value of $\leq 0.01$. The range of $\% \mathrm{P}$ in this study was $1.7-77.3 \%$ (mean of $61.4 \%$ ). Thrity-six per cent of the variation in $\% \mathrm{P}$ was explained by individual-level differences (adjusted $R^{2}$ measure).

Outside of $\mathrm{pH}$ and PMI, which for reasons described previously were analysed separately, the most important covariates were brain region (explained $12.4 \%$ of the variation in $\% \mathrm{P}, p=3.6 \times 10^{-53}$ ), followed by brain bank (explained an additional $4.7 \%, p=9.6 \times 10^{-6}$ ), and then RIN (explained an additional 2.7\%, $p=9.1 \times 10^{-05}$ ). Age, gender, and cause of death together explained an additional $2.2 \%$ of the variation. The effect of brain region on \% P was most obvious when comparing CRBL and WHMT. CRBL showed highest $\% \mathrm{P}($ mean $=68 \%)$ whereas WHMT showed the lowest $\% \mathrm{P}($ mean $=57.5 \%)($ Fig. 3$)$.

$\mathrm{pH}$ explained $12.0 \%$ of the variation in $\% \mathrm{P}$ from the MRC-UK brain bank $\left(p=2.3 \times 10^{-9}\right)$. (Fig. 4). However, as with RIN, this correlation was highly dependent on the six individuals with low $\mathrm{pH}<5.90$. When these samples were excluded from the analysis no significant correlation was obtained.

Finally, no significant correlation was found between PMI and $\% \mathrm{P}$, either for the MRC-UK dataset or the SHRI-USA dataset.

Moreover, no difference was observed in the expected nucleotide lengths following cDNA preparation $(\sim 200-400$ nt) and cRNA production (ranges from 200 to $2000 \mathrm{nt}$ ) between different samples with different RIN values (as shown by Agilent Bioanalyser profiles).

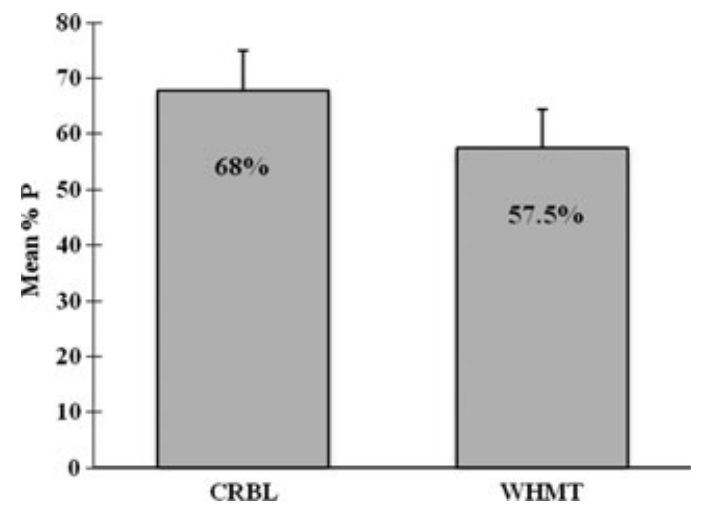

Fig. 3 Bar chart to show variation in \%P by brain region (CRBL, WHMT).This graph shows the different performance of samples from specific brain regions on the array. These results are highly significant $p$-values $\left(1.3 \times 10^{-24}\right)$. The heights of the bars represent the mean. The error bars represent the SEM. 


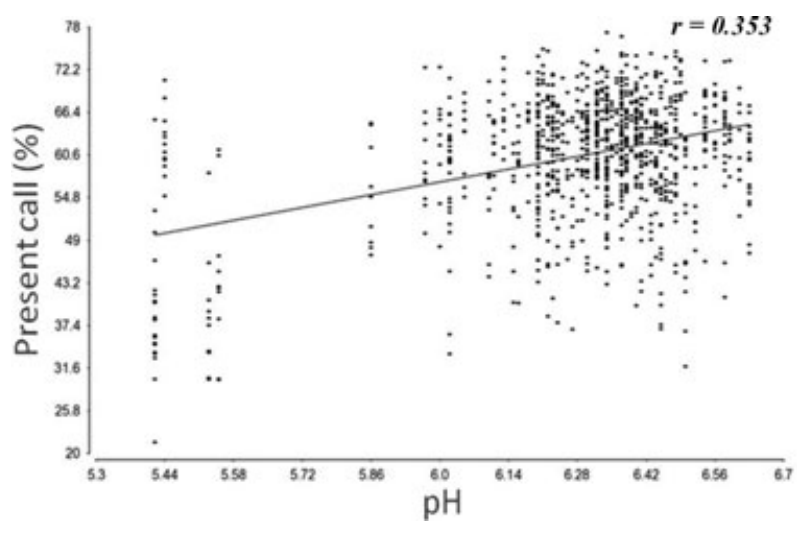

Fig. 4 Scatter plot for total RNA samples with linear regression line of Present call $(\% \mathrm{P})$ for $\mathrm{pH}$. Scatter plot shows that $\mathrm{pH}$ significantly explains $12.0 \%$ of the variation in \% $\mathrm{P}\left(p=2.3 \times 10^{-9}, r=0.353\right)$, including samples with low $\mathrm{pH}$ values. Low $\mathrm{pH}$ values are driving the regression analysis.

The reproducibility of array data using QuantiGene, a PCR-independent platform to confirm expression results QuantiGene is a technique for mRNA expression quantification. Its strength is that it is a non-PCR based technique that is therefore not subject to the systematic biases that PCR could create when applied to degraded RNA. This technique has the potential to be used in place of qRT-PCR (Canales et al. 2006; Hall et al. 2011).

We validated the exon array results in a subset of 12 individuals by comparing the normalised mRNA expression level for three genes (LRRK2, SCN8A and MAPT), in four brain regions (CRBL, OCTX, PUTM and WHMT). We observed a similar regional pattern of expression across the

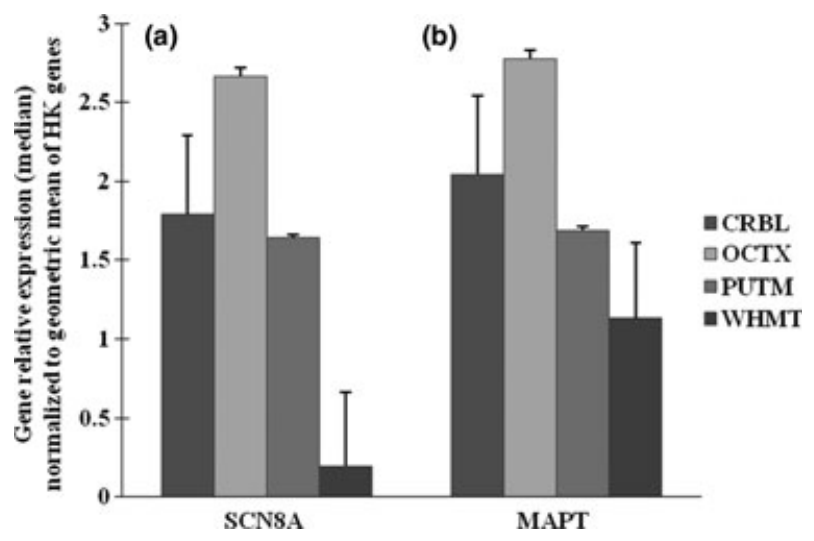

Fig. 5 QuantiGene validation of microarray expression data. (a) SCN8A expression level between different regions. The graph shows high expression in OCTX compare with other regions. It is clear that this gene in mostly not expressed in WHMT region (expression level close to zero) also it presenting large error bar. (b) MAPT, showing higher expression in OCTX compare with other brain regions. These results confirm the array data with significant $p$-values of $<0.01$. The expression level is presenting the mean and the error bars is SEM.

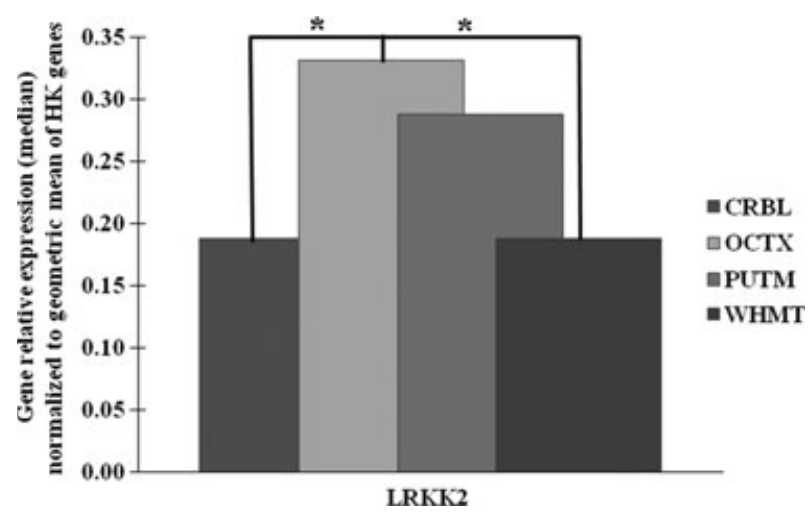

Fig. 6 QuantiGene validation of microarray expression data for LRRK2 expression level in different regions. The graph shows higher expression in OCTX compare with other regions. Wilcoxon-signed rank test was performed and these results confirm the difference between regions in array data is significant. The expression level is presenting the median and the stars indicating the significant difference in expression with $p$-values of $<0.01$. In this case, as LRRK2 expression level was very low, it was unreliable to use SEM and Wilcoxon's test was performed using the median values.

two platforms for both high (SCN8A and MAPT) (Fig. 5) and low expression transcripts in brain (LRRK2) (Fig. 6).

Furthermore, there was an excellent correlation in fold change $\left(r^{2}=0.855\right)$ at both the gene and exon expression levels and signal intensity values $\left(r^{2}=0.91\right)$ in the four brain regions we studied.

\section{Discussion}

To our knowledge, the UK Human Brain Expression Consortium data set is the largest control brain microarray data set generated to date. It is based on the analysis of tissue samples from 13 different CNS regions originating from 137 individuals and containing 2318 processed samples. The main goal of this project is to build a large reference database for $\mathrm{eQTL}$ and $\mathrm{SQTL}$ analysis.

This study showed considerable variation in RIN values among RNA samples. Sixty-seven per cent of the variation resides in differences among extractions from the same tissue blocks and most of the remaining variation is unexplained by the available covariate information. We found that $\mathrm{pH}$ is the most important post-mortem factor influencing RIN-based RNA integrity, a result consistent with previous studies (Hardy et al. 1985; Mexal et al. 2006; Chevyreva et al. 2008; Monoranu et al. 2009; Durrenberger et al. 2010). Samples with very low $\mathrm{pH}$ values (ranging from 5.42 to 5.90 ) were responsible for the positive correlation seen between $\mathrm{pH}$ and \% (and also RIN). However, when these low $\mathrm{pH}$ samples were removed from the analysis we no longer observed any significant correlation. This may in part explain contradictory observations 
regarding the effect of $\mathrm{pH}$ on RNA integrity and sample performance on arrays (Hardy et al. 1985; Monoranu et al. 2009; Birdsill et al. 2010) and confirms the findings of a recent, but smaller study on control brain tissue (Tomita et al. 2004; Sherwood et al. 2011).

We found our array-based expression data to be reliably validated by the QuantiGene PCR-independent method, when tested on two high expression genes (MAPT and SCN8A) and one low expression gene (LRRK2). However, the performance quality of the array, as defined by $\% \mathrm{P}$, was not profoundly affected by age, gender, region, PMI, RIN and cause of death. This confirms findings from previous studies on much smaller sample sizes (Tomita et al. 2004; Birdsill et al. 2010; Durrenberger et al. 2010). We found that only $2.7 \%$ of the variation in \% $\mathrm{P}$ was explained by RIN. Indeed, 80 RNA samples with undetectable RINs performed well on the arrays with $\% \mathrm{P}$ values ranging from 45 to $76 \%$. Thus, we found RIN to be a poor predictor of array quality performance even at the low end of the RIN scale. Furthermore, the latter was confirmed since the cDNA and cRNA length synthesis was not affected by the wide range of RIN values (from 2 to 7 ) in our array experiments. The robust performance of the Affymetrix Exon arrays in the face of degraded RNA may be due to recent changes to the RNA amplification process. In keeping with the manufacturer's instructions, this was performed using the Ambion ${ }^{\circledR}$ WT Expression kit, which uses both non-polyA and polyA-based mRNA priming for first strand cDNA synthesis. This meant that RNA amplification did not require an intact polyA tail. In addition, increasing the quantity of the starting material of RNA from 500 to $750 \mathrm{ng}$ improved the array performance.

Through the analysis of this observational study, we experienced different limitations. For example, we had expected that cause of death would greatly influence both RIN-based RNA quality and \%P-based array quality, but cause of death only explained $1.9 \%$ of variation in RIN and we did not find any significant relationship with \%P. It may be that cause of death is an imperfect reflection of the true medical and drug treatment history of the individual, and that access to that history, were it available, would reveal other factors of greater relevance. Likewise, in the range of 28 $114 \mathrm{~h}$ PMI did not affect on either RIN or \%P, nor could we see a loss of RIN-based RNA quality over the $1-5 \mathrm{~h}$ range. It remains possible that there may be selective loss of RNA within an hour because the half-life of some mRNA species has been reported to be as short as $15 \mathrm{~min}$, while others may be as long as 22 days depending on the tissue type and storage conditions (Ross 1995; Barrachina et al. 2006; Bahar et al. 2007; Beach et al. 2008; Vennemann and Koppelkamm 2010). This issue has been studied in detail for a range of PMIs by Harrison et al. 1995 and confirmed by a more recent study by Tomita et al. (2004). The same conclusion was made that PMI had a limited effect on mRNA (Harrison et al. 1995; Tomita et al. 2004).
Furthermore, there is a possibility that samples with the shortest PMIs (1-2.5 h) within the SHRI-USA sample set may originate from those individuals who suffered longer agonal states prior to death and agonal stress has been shown to affect gene expression differently in different brain regions (Li et al. 2007). Other factors may contribute to this such as intermittent edge effects, especially on small samples during dissecting and tissue handling procedures.

Finally, we note that our brain samples have been derived from only two sources: one was a rapid death brain bank with long post-mortem intervals and the other specialises in obtaining very short post-mortem intervals. Both brain banks had separately optimised their protocols to facilitate gene expression studies and this may limit the generalisability of these conclusions to tissue collected in other ways.

These results are important for several reasons. Firstly, they confirm the practical feasibility of using post-mortem control brain tissue to study the transcriptome of the human brain by array technology. Secondly, they show that microarrays can give reliable results over a wide range of RIN numbers (1-8.5) and $\mathrm{pH}$ measurements with drop off in array validity only being observed below brain $\mathrm{pH}$ 5.9. Thirdly, they show that the results from Affymetrix exon arrays are reproducible by other technologies, making it possible for database users to use the data generated with confidence.

Furthermore, this study is the first step of an ongoing multi-regional human brain expression project that has been established to build an open-access database of identified genome-wide genetic variability in relation with gene eQTLs and sQTL as well as for detailed expression analysis (Hardy et al. 2009). We hope this will move the field forward in our understanding of the underlying molecular mechanisms of complex neurological and psychiatric diseases, and will support the neuroscience community with a resource which will bring functional insights.

\section{Acknowledgements}

This work was supported by the MRC through the MRC Sudden Death Brain Bank (CS) and through MRC Project Grants (John Hardy and Michael Weale) and Training Fellowship (Mina Ryten) schemes. Daniah Trabzuni was supported by King Faisal Specialist Hospital and Research Centre, Saudi Arabia. We would like to thank AROS Applied Biotechnology AS company laboratories. We would also like to specially thank Jane Ramsey, Geoff Scopes and Wilson Lew (Affymetrix) for their valuable input. The authors have no conflicting financial interests.

\section{References}

Arikawa E., Sun Y., Wang J., Zhou Q., Ning B., Dial S. L., Guo L. and Yang J. (2008) Cross-platform comparison of SYBR Green realtime PCR with TaqMan PCR, microarrays and other gene expression measurement technologies evaluated in the MicroArray Quality Control (MAQC) study. BMC Genomics 9, 328. 
Bahar B., Monahan F. J., Moloney A. P., Schmidt O., MacHugh D. E. and Sweeney T. (2007) Long-term stability of RNA in post-mortem bovine skeletal muscle, liver and subcutaneous adipose tissues. BMC Mol. Biol. 8, 108.

Barrachina M., Castano E. and Ferrer I. (2006) TaqMan PCR assay in the control of RNA normalization in human post-mortem brain tissue. Neurochem. Int. 49, 276-284.

Beach T. G., Sue L. I., Walker D. G., Roher A. E., Lue L., Vedders L., Connor D. J., Sabbagh M. N. and Rogers J. (2008) The Sun Health Research Institute Brain Donation Program: description and experience, 1987-2007. Cell Tissue Bank 9, 229-245.

Birdsill A. C., Walker D. G., Lue L., Sue L. I. and Beach T. G. (2010) Postmortem interval effect on RNA and gene expression in human brain tissue. Cell Tissue Bank [Epub ahead of print].

Burke W. J., O’Malley K. L., Chung H. D., Harmon S. K., Miller J. P. and Berg L. (1991) Effect of pre- and postmortem variables on specific mRNA levels in human brain. Brain Res. Mol. Brain Res. 11, 37-41.

Canales R. D., Luo Y. and Willey J. C. et al. (2006) Evaluation of DNA microarray results with quantitative gene expression platforms. Nat. Biotechnol. 24, 1115-1122.

Chervoneva I., Li Y., Schulz S., Croker S., Wilson C., Waldman S. A. and Hyslop T. (2010) Selection of optimal reference genes for normalization in quantitative RT-PCR. BMC Bioinform. 11, 253.

Chevyreva I., Faull R. L., Green C. R. and Nicholson L. F. (2008) Assessing RNA quality in postmortem human brain tissue. Exp. Mol. Pathol. 84, 71-77.

Chomczynski P. and Sacchi N. (1987) Single-step method of RNA isolation by acid guanidinium thiocyanate-phenol-chloroform extraction. Anal. Biochem. 162, 156-159.

Coulson D. T., Brockbank S., Quinn J. G., Murphy S., Ravid R., Irvine G. B. and Johnston J. A. (2008) Identification of valid reference genes for the normalization of RT qPCR gene expression data in human brain tissue. BMC Mol. Biol. 9, 46.

Durrenberger P. F., Fernando S. and Kashefi S. N. et al. (2010) Effects of antemortem and postmortem variables on human brain mRNA quality: a BrainNet Europe study. J. Neuropathol. Exp. Neurol. 69, 70-81.

Gilbert J. M., Brown B. A., Strocchi P., Bird E. D. and Marotta C. A. (1981) The preparation of biologically active messenger RNA from human postmortem brain tissue. J. Neurochem. 36, 976-984.

Glanzer J. G., Haydon P. G. and Eberwine J. H. (2004) Expression profile analysis of neurodegenerative disease: advances in specificity and resolution. Neurochem. Res. 29, 1161-1168.

Glasel J. A. (1995) Validity of nucleic acid purities monitored by 260nm/280nm absorbance ratios. BioTechniques 18, 62-63.

Hall J. S., Leong H. S. and Armenoult L. S. et al. (2011) Exon-array profiling unlocks clinically and biologically relevant gene signatures from formalin-fixed paraffin-embedded tumour samples. $\mathrm{Br}$. J. Cancer 104, 971-981.

Hardy J. A., Wester P., Winblad B., Gezelius C., Bring G. and Eriksson A. (1985) The patients dying after long terminal phase have acidotic brains; implications for biochemical measurements on autopsy tissue. J. Neural Transm. 61, 253-264.

Hardy J., Trabzuni D. and Ryten M. (2009) Whole genome expression as a quantitative trait. Biochem. Soc. Trans. 37, 1276-1277.

Harrison P. J., Heath P. R., Eastwood S. L., Burnet P. W., McDonald B. and Pearson R. C. (1995) The relative importance of premortem acidosis and postmortem interval for human brain gene expression studies: selective mRNA vulnerability and comparison with their encoded proteins. Neurosci. Lett. 200, 151-154.

Imbeaud S., Graudens E., Boulanger V., Barlet X., Zaborski P., Eveno E., Mueller O., Schroeder A. and Auffray C. (2005) Towards standardization of RNA quality assessment using user-independent classifiers of microcapillary electrophoresis traces. Nucleic Acids Res. 33, e56.

de Jonge H. J., Fehrmann R. S. and de Bont E. S. et al. (2007) Evidence based selection of housekeeping genes. PLOS ONE 2, e898.

Li J. Z., Meng F. and Tsavaler L. et al. (2007) Sample matching by inferred agonal stress in gene expression analyses of the brain. BMC Genomics 8, 336.

Lockstone H. E. (2011) Exon array data analysis using Affymetrix power tools and R statistical software. Brief Bioinform, [Epub ahead of print].

Mexal S., Berger R., Adams C. E., Ross R. G., Freedman R. and Leonard S. (2006) Brain $\mathrm{pH}$ has a significant impact on human postmortem hippocampal gene expression profiles. Brain Res. 1106, 1-11.

Millar T., Walker R., Arango J. C., Ironside J. W., Harrison D. J., MacIntyre D. J., Blackwood D., Smith C. and Bell J. E. (2007) Tissue and organ donation for research in forensic pathology: the MRC Sudden Death Brain and Tissue Bank. J. Pathol. 213, 369-375.

Monoranu C. M., Apfelbacher M. and Grunblatt E. et al. (2009) pH measurement as quality control on human post mortem brain tissue: a study of the BrainNet Europe consortium. Neuropathol. Appl. Neurobiol. 35, 329-337.

Myers A. J., Gibbs J. R. and Webster J. A. et al. (2007) A survey of genetic human cortical gene expression. Nat. Genet. 39, 1494-1499.

Ross J. (1995) mRNA stability in mammalian cells. Microbiol. Rev. 59, 423-450.

Sajdel-Sulkowska E. M., Majocha R. E., Salim M., Zain S. B. and Marotta C. A. (1988) The postmortem Alzheimer brain is a source of structurally and functionally intact astrocytic messenger RNA. J. Neurosci. Methods 23, 173-179.

Schroeder A., Mueller O. and Stocker S. et al. (2006) The RIN: an RNA integrity number for assigning integrity values to RNA measurements. BMC Mol. Biol. 7, 3.

Sherwood K. R., Head M. W., Walker R., Smith C., Ironside J. W. and Fazakerley J. K. (2011) RNA integrity in post-mortem human vCJD and control brain tissue. Neuropathol. Appl. Neurobiol., 37, 633-642.

Stan A. D., Ghose S., Gao X. M., Roberts R. C., Lewis-Amezcua K., Hatanpaa K. J., Tamminga C. A. (2006) Human postmortem tissue: what quality markers matter? Brain Res. 1123, 1-11.

Tomita H., Vawter M. P. and Walsh D. M. et al. (2004) Effect of agonal and postmortem factors on gene expression profile: quality control in microarray analyses of postmortem human brain. Biol. Psychiatry 55, 346-352.

Vennemann M. and Koppelkamm A. (2010) mRNA profiling in forensic genetics I: possibilities and limitations. Forensic Sci. Int. 203, $71-75$.

Weis S., Llenos I. C., Dulay J. R., Elashoff M., Martinez-Murillo F. and Miller C. L. (2007) Quality control for microarray analysis of human brain samples: The impact of postmortem factors, RNA characteristics, and histopathology. J. Neurosci. Methods 165, 198-209. 